\title{
Remoción de iones hierro(II) en medio acuoso con una toba vítrea natural
}

\section{Removal of iron(II) ions in aqueous medium using a natural Cuban vitreous tuff}

\author{
Miguel Garrido-Rodríguez ${ }^{1 *}$, Mercedes Eulalia Sosa-Martínez ${ }^{2}$, María Caridad \\ Ramírez-Pérez ${ }^{2}$, Pavel Figueroa-Ramírez ${ }^{1}$, Ezequiel Fonseca-González ${ }^{1}$ \\ ${ }^{1}$ Universidad Tecnológica de La Habana, Cuba \\ ${ }^{2}$ Universidad de Moa, Holguín, Cuba \\ *Autor para la correspondencia: magarrido@quimica.cujae.edu.cu
}

\section{Resumen}

Se evaluó la capacidad de absorción en la remoción de iones hierro(II) en una fase líquida, de una toba vítrea cubana en estado natural del yacimiento Magueyal. Se prepararon disoluciones moduladas y de un residual líquido generado durante la limpieza química de las superficies internas de las calderas en las termoeléctricas, el cual presenta altas concentraciones de iones hierro (II) en su composición. La cinética del proceso se describió por el modelo de pseudosegundo orden, donde la ecuación isoterma de adsorción de Freundlich resultó con el mejor ajuste. Se alcanza una capacidad máxima de adsorción igual a $34,4 \mathrm{mg} / \mathrm{g}$ de iones hierro(II) en la toba vítrea.

Palabras clave: toba vítrea; adsorción; modelo cinético; isoterma de adsorción; capacidad de adsorción.

\section{Abstract}

This study assessed the absorption capacity in removing iron (II) ions in a liquid phase of a Cuban glassy tuff in natural state from Magueyal deposit. Modulated solutions and a liquid residual generated during the chemical cleaning of the internal surfaces of the boilers in the thermoelectric plants were prepared, showing high concentrations of iron(II) ions in its composition. The pseudo second order model was used to determine the 
kinetics of the process, where Freundlich's isothermal adsorption equation was the best fit, and a maximum adsorption capacity equal to $34.4 \mathrm{mg} / \mathrm{g}$ of iron (II) ions was reached.

Keywords: glassy tuff; adsorption; kinetic model; adsorption isotherm; adsorption capacity.

\section{INTRODUCCIÓN}

El agua es esencial para la humanidad, por lo que su uso racional y el control de agentes contaminantes en los residuales de procesos industriales constituyen objetivos de la investigación científica. En particular, el tratamiento de los residuales líquidos antes de ser vertidos a los cuerpos de agua naturales para eliminar la mayor cantidad de contaminantes constituye una de las vías para evitar la contaminación ambiental.

Entre los métodos aplicados, la adsorción mediante materiales de bajo costo como las zeolitas, las tobas vítreas, las arcillas y bioadsorbentes constituyen una alternativa viable para la recuperación de iones metálicos (Albadarin et al. 2012; Batista 2016; Córdova 2015; Ghassabzadeha et al. 2010; Niboua et al. 2010; Keng et al. 2014).

Investigaciones en las que se han usado minerales activados (MachadoInfante, Ramírez-Caballero y Barajas-Meneses 2016) demuestran que en minerales la adsorción de hierro(II) a partir de soluciones acuosas se describe mediante la ecuación isoterma de adsorción de Langmuir y el modelo cinético de pseudosegundo orden. De igual modo, Vera-Alatrista (2006) demuestra la posibilidad del uso de la adsorción para disminuir la presencia de contaminantes en aguas residuales, entre ellos hierro(II).

La limpieza química de las calderas en las termoeléctricas constituye una fuente de residuales líquidos con alta concentración de metales, incluido el hierro(II).

Esta investigación tuvo el objetivo de analizar la capacidad de adsorción de la toba vítrea del yacimiento Magueyal de Santiago de Cuba, como adsorbente del hierro(II) del residual líquido que se genera durante la limpieza química de las superficies internas de las calderas en las termoeléctricas. 


\section{MATERIALES Y MÉTODOS}

\subsection{Características del mineral adsorbente}

El mineral arcilloso, sin tratamiento previo o activación, inicialmente se sometió a trituración y clasificación, seleccionando la muestra con una granulometría de $0,25 \mathrm{~mm}$. La caracterización química y superficial de este material se expone en investigaciones precedentes (Blanco-Flores et al. 2009; Fernández-Hechevarría et al. 2016). El mineral posee propiedades adsorbentes y se caracteriza por el alto contenido de óxidos de silicio y aluminio (Tabla 1).

Tabla 1. Composición química del mineral Magueyal

\begin{tabular}{ccccccccc}
\hline Compuesto & $\mathbf{K}_{\mathbf{2}} \mathbf{O}$ & $\mathbf{F e}_{2} \mathbf{O}_{\mathbf{3}}$ & $\mathbf{N a}_{\mathbf{2}} \mathbf{O}$ & $\mathbf{C a O}$ & $\mathbf{S i O}_{2}$ & $\mathbf{M g O}$ & $\mathbf{T i O}_{2}$ & $\mathbf{A l}_{2} \mathbf{O}_{3}$ \\
\hline $\begin{array}{c}\text { Porcentaje } \\
(\%)\end{array}$ & 2,36 & 3,1 & 1,11 & 1,56 & 68,12 & 0,86 & 0,2 & 11,51
\end{tabular}

En la etapa inicial se investigó la adsorción de iones hierro(II) en soluciones moduladas, preparadas usando sulfato de hierro(II) hexahidratado puro para análisis, con concentraciones similares a los residuales reales de la limpieza química en las termoeléctricas. El estudio incluyó la adsorción conjunta con iones cobre(II) para analizar su influencia en la remoción de iones hierro(II).

En la segunda etapa se analizó la capacidad de adsorción de la toba vítrea con la utilización de soluciones residuales reales obtenidas de la etapa de decapado ácido en una caldera, donde la concentración de iones hierro(II) fue de $5,1 \mathrm{~g} / \mathrm{L}$. Para disminuir la acidez de la solución se realizó un tratamiento con hidróxido de sodio hasta que el $\mathrm{pH}$ del residual fuese igual a siete unidades y la concentración de iones hierro(II) de $14,8 \mathrm{mg} / \mathrm{L}$, superior al límite máximo permisible, según la norma NC 521: 2007.

\subsection{Métodos de estudio cinético}

Existen varios modelos matemáticos empíricos que permiten obtener los parámetros cinéticos de adsorción con los cuales es posible describir el fenómeno físico o químico que tiene lugar. Entre estos se encuentran: modelo de pseudoprimer orden, pseudosegundo orden, segundo orden y difusión intraparticular (Azizian 2004).

La adsorción en la interface sólido-líquido en un sistema perfectamente mezclado es descrita, generalmente, por las etapas siguientes:

Etapa 1. Difusión del soluto desde el seno de la fase líquida hacia la película líquida que rodea el adsorbente. 
Etapa 2. Difusión a través de la película líquida que rodea las partículas del adsorbente.

Etapa 3. Difusión en los poros del adsorbente o difusión intraparticular.

Etapa 4. Adsorción en los sitios activos del material.

Se considera que la velocidad del proceso está controlada por las etapas 2 y 3, difusión externa y la difusión intraparticular, respectivamente.

\subsection{Modelo de pseudoprimer orden (modelo de Lagergren)}

Este modelo se usa frecuentemente para adsorbentes homogéneos y adsorción física, la velocidad de adsorción es proporcional a la concentración de soluto y está descrito por la ecuación 1.

$q_{t}=q_{e} \cdot\left(1-e^{k_{L} \cdot t}\right)$

Donde:

$\mathrm{q}_{\mathrm{t}}$ : cantidad del ión adsorbido en un tiempo $\mathrm{t}(\mathrm{mol} / \mathrm{g})$.

$\mathrm{q}_{\mathrm{e}}$ : cantidad del ión adsorbido en el equilibrio ( $\left.\mathrm{mol} / \mathrm{g}\right)$.

$\mathrm{k}_{\mathrm{L}}$ : constante de adsorción de Lagergren $\left(\mathrm{min}^{-1}\right)$.

t: tiempo $(\min )$.

\subsection{Modelo de pseudosegundo orden}

En el modelo de pseudosegundo orden se asume que la velocidad del paso controlante puede estar dada por la quimisorción, que involucra la fuerza de valencia a través del intercambio de electrones entre el adsorbato y el adsorbente (Plazinski, Dziuba y Rudzinski 2013). El modelo está representado por la ecuación 2.

$$
\frac{t}{q_{t}}=\frac{q_{e}^{2} \cdot k_{2} \cdot t}{1+q_{e} \cdot k_{2} \cdot t}
$$

Donde:

$\mathrm{q}_{\mathrm{t}}$ : cantidad del ión adsorbido en un tiempo t $(\mathrm{mol} / \mathrm{g})$

$\mathrm{q}_{\mathrm{e}}$ : cantidad del ión adsorbido en el equilibrio $(\mathrm{mol} / \mathrm{g})$

$\mathrm{k}_{2}$ : constante de velocidad de pseudosegundo orden para el proceso de adsorción $(\mathrm{g} / \mathrm{mol} * \mathrm{~min})$ 


\subsection{Modelo de segundo orden}

Este es un modelo muy utilizado en quimisorción sobre materiales altamente heterogéneos y está representado por la ecuación 3.

$$
q_{t}=\frac{1}{b} \cdot(1-a \cdot b \cdot t)
$$

Donde:

$\mathrm{q}_{\mathrm{t}}$ : cantidad del ión adsorbido en un tiempo $\mathrm{t}(\mathrm{mol} / \mathrm{g})$

a: constante de adsorción del ión ( $\mathrm{mol} / \mathrm{g}$ )

b: constante de desorción $(\mathrm{g} / \mathrm{mol} * \min )$

\subsection{Modelo de la difusión intraparticular}

La velocidad de adsorción se determina usualmente con el uso del modelo de difusión intraparticular. Lo cual se logra graficando la cantidad de iones adsorbidos por peso unitario de adsorbente $\left(\mathrm{q}_{\mathrm{t}}\right)$, en función del tiempo $(\mathrm{t})$. La adsorción en la interface sólido-líquido, en un sistema perfectamente mezclado, es descrita normalmente en tres pasos:

Paso 1: Difusión a través de la película que rodea las partículas del adsorbente, llamada también difusión externa.

Paso 2: Difusión en los poros del adsorbente o difusión intraparticular.

Paso 3: Adsorción en los sitios activos del material.

Se considera que la velocidad controlante de los pasos 1 y 2 viene dada por la transferencia de masa externa y la difusión intraparticular, respectivamente. El paso 3 generalmente es el más rápido de todos.

La constante de difusión intraparticular (superficie interna y difusión en el poro) puede ser calculada a través de la ecuación 4.

$q_{t}=k_{i} \cdot t^{0,5}+c$

Donde:

$\mathrm{q}_{\mathrm{t}}$ : cantidad del ión adsorbido en un tiempo $\mathrm{t}(\mathrm{mol} / \mathrm{g})$.

$\mathrm{k}_{\mathrm{d}}$ : constante de velocidad intraparticular $\left(\mathrm{mol} / \mathrm{g}^{\mathrm{x}} \mathrm{min}^{0,5}\right)$.

c: intercepto de la línea recta (proporcional al grosor de la capa límite) (mol/g). 


\subsection{Isotermas de adsorción}

El estudio de adsorción puede realizarse mediante la identificación de la forma y la curvatura de las isotermas de adsorción y mediante su formulación matemática. La forma de las isotermas depende, en gran medida, del tipo de adsorbente y el adsorbato, por tanto, las mismas serán diferentes para cada tipo de estos.

Las isotermas de adsorción experimentales se dividen, por lo general, en seis categorías, según la clasificación de la IUPAC, pero solo se utilizan las cinco primeras (Figura 1).

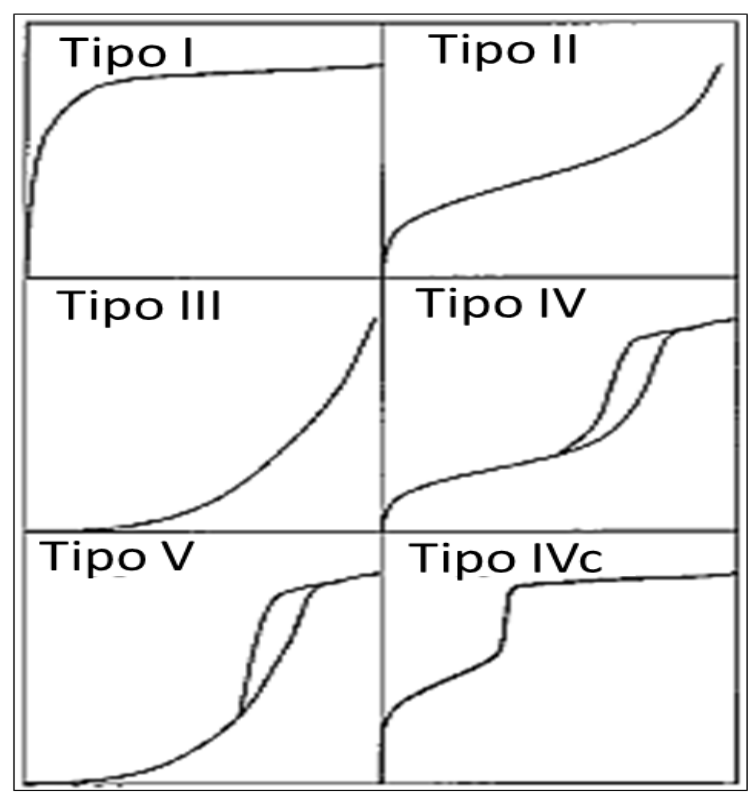

Figura 1. Clasificación de las isotermas experimentales de adsorción.

Para el análisis de los datos experimentales se han desarrollado diversos modelos matemáticos, siendo los más utilizados el de Langmuir, Freundlich y Langmuir-Freundlich. Aunque existen otros modelos como el de RedlinchPeterson, Toth, Tempkin, entre otros.

\subsection{Isoterma de Langmuir}

Este modelo considera que la superficie está idealmente limpia y homogénea. La adsorción ocurre en sitios específicos del adsorbente y todos son idénticos. Solo un adsorbato ocupa cada sitio y una vez formada la monocapa cesa la adsorción. El modelo puede ser descrito por la ecuación 5.

Qads $=\frac{K_{L} *\left(N_{m} * C_{e q}\right)}{1+K_{L} * \operatorname{Ceq}}$ 
Donde:

Qads: cantidad de soluto adsorbido por peso unitario de adsorbente $(\mathrm{mol} / \mathrm{g})$.

Nm: capacidad máxima de adsorción (mol/g).

$\mathrm{K}_{\mathrm{L}}$ : constante de saturación (mol/L).

$\mathrm{C}_{\text {eq }}$ : concentración del adsorbato en la disolución en el equilibrio ( $\mathrm{mol} / \mathrm{L}$ ).

Las características esenciales del modelo de Langmuir pueden ser expresadas en términos del factor de separación $\left(R_{L}\right)$, el cual está definido por la ecuación 6.

$R_{L}=\frac{1}{1+K_{L} * \operatorname{Ceq}}$

De acuerdo con el valor de $\mathrm{R}_{\mathrm{L}}$ la forma de la isoterma puede ser interpretada de la siguiente forma:

$\mathrm{R}_{\mathrm{L}}>1$ : adsorción no favorable

$\mathrm{R}_{\mathrm{L}}=1$ : adsorción lineal

$0<\mathrm{R}_{\mathrm{L}}<1$ : adsorción favorable

$\mathrm{R}_{\mathrm{L}}=0$ : adsorción irreversible.

\subsection{Isoterma de Freundlich}

Este modelo fue establecido empíricamente y se basa en que la adsorción es de naturaleza física, no hay asociación de las moléculas adsorbidas y la superficie del adsorbente es heterogénea. Es un modelo útil para determinar la adsorción en soluciones líquidas, y riguroso para los casos que ocurren en superficies heterogéneas. El modelo clásico de Freundlich está descrito por la ecuación 7.

Qads $=k_{F} * C_{e q}{ }^{1 / n}$

Donde:

Qads: cantidad de soluto adsorbido por peso unitario de adsorbente (mol/g).

1/n: coeficiente de Freundlich $(1<n<10)$. 
$\mathrm{k}_{\mathrm{F}}$ : constante de Freundlich $(\mathrm{mol} / \mathrm{g})$.

$\mathrm{C}_{\mathrm{eq}}$ : concentración del adsorbato en la disolución en el equilibrio ( $\left.\mathrm{mol} / \mathrm{L}\right)$.

\subsection{Isoterma de Langmuir-Freundlich}

El modelo de Langmuir-Freundlich es una combinación de los mismos, por lo que representa una adsorción mediante una combinación de mecanismos sobre materiales heterogéneos. La ecuación 8 describe este modelo.

$Q_{a d s}=\frac{K * C_{e q}{ }^{1 / n}}{1+b * c_{e q}^{1 / n}}$

Donde:

Qads: cantidad de soluto adsorbido por peso unitario de adsorbente $(\mathrm{mol} / \mathrm{g})$

$1 / n$ : coeficiente de Freundlich $(1<n<10)$

$\mathrm{K}, \mathrm{b}$ : constantes empíricas del modelo de Langmuir

$C_{\text {eq }}$ : concentración del adsorbato en la disolución en el equilibrio ( $\left.\mathrm{mol} / \mathrm{L}\right)$.

\section{RESULTADOS Y DISCUSIÓN}

\subsection{Estudio cinético del proceso de adsorción}

El análisis de los resultados del estudio cinético de la adsorción mostró el incremento progresivo de la Qads hasta los $80 \mathrm{~min}$ en que se alcanza el equilibrio con $0,047 \mathrm{mg} / \mathrm{L}$ de hierro(II). La utilización de los modelos de pseudoprimer orden, pseudosegundo orden y segundo orden (Tabla 2) permitió comprobar que el proceso es descrito por el modelo de pseudoprimer orden, con un coeficientes de correlación $\left(R^{2}=0,9771\right)$ (Toor y Jin 2012).

Tabla 2. Modelos cinéticos en la adsorción de hierro (II) en la toba vítrea

\begin{tabular}{ccc}
\hline Modelo cinético & \multicolumn{2}{c}{ Parámetros } \\
\hline \multirow{2}{*}{ Pseudoprimer orden } & $\mathrm{K}_{\mathrm{L}}\left(\mathrm{min}^{-1}\right)$ & 0,452 \\
& $\mathrm{R}^{2}$ & 0,9771 \\
Pseudosegundo orden & $\mathrm{k}(9 / \mathrm{mol} \cdot \min )$ & 1,037 \\
& $\mathrm{R}^{2}$ & 0,9639 \\
Segundo orden & $\mathrm{a}$ & 0,035 \\
& $\mathrm{~b}$ & 78,74 \\
& $\mathrm{R}^{2}$ & 0,847
\end{tabular}


El proceso de transferencia de hierro(II) desde la fase líquida a la sólida tiene lugar en tres etapas:

1. La difusión de los iones a través de la película líquida que rodea al adsorbente

2. La difusión intraparticular

3. La adsorción de los sitios activos.

Al ajustar el modelo de difusión lineal se comprobaron las etapas, como se muestra en la Figura 2, siendo la difusión a través de la capa de líquido que rodea al adsorbente la que limita el proceso.

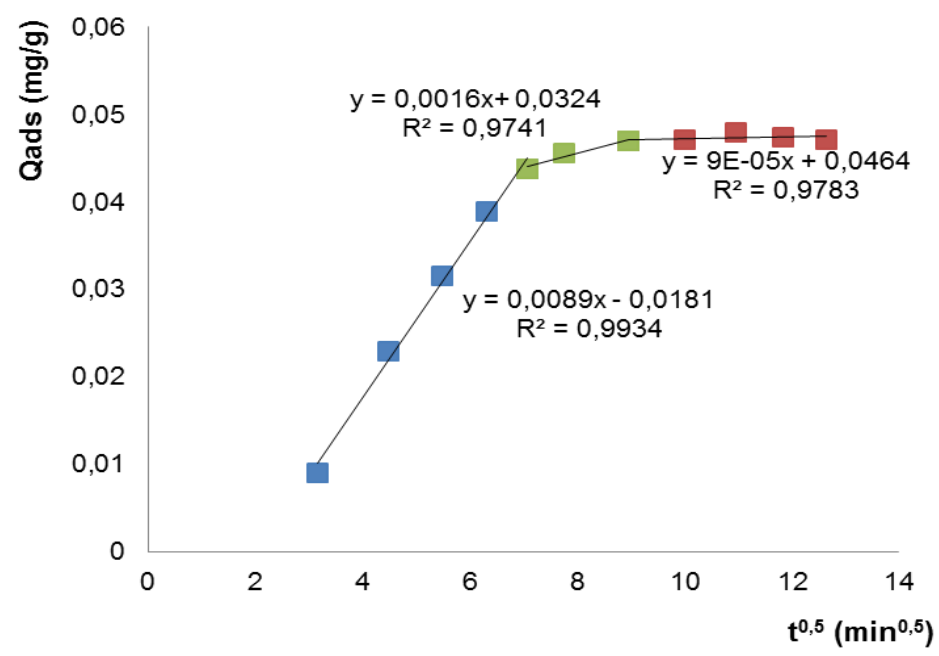

Figura 2. Difusión intraparticular del hierro(II) en el mineral Magueyal en estado natural.

Teniendo en cuenta este comportamiento representado en la primera y segunda zona lineal, difusión intraparticular y difusión a través de la película de líquido, respectivamente, son influyentes en la velocidad del proceso de remoción del hierro(II). Los parámetros cinéticos de las etapas del proceso se indican en la Tabla 3. 
Tabla 3. Parámetros de los modelos cinéticos en la adsorción de hierro(II) en el mineral de Magueyal en estado natural

\begin{tabular}{ccc}
\hline Etapas & \multicolumn{2}{c}{ Parámetros del modelo } \\
\hline $\begin{array}{c}\text { Difusión a través de la } \\
\text { película líquida que } \\
\text { rodea al sólido }\end{array}$ & Pendiente & 0,0089 \\
& Intercepto & $-0,0181$ \\
Difusión intraparticular & $\mathrm{R}^{2}$ & 0,9934 \\
& Pendiente & 0,0016 \\
Adsorción en los sitios & Intercepto & 0,0324 \\
activos & $\mathrm{R}^{2}$ & 0,9741 \\
& Pendiente & 0,00009 \\
\hline
\end{tabular}

\subsection{Isotermas de adsorción}

Los resultados experimentales de la adsorción del hierro(II) en la toba vítrea (Figura 3) mostraron el incremento de la cantidad de soluto adsorbido por unidad de peso de adsorbente (Qads), en función de la concentración de los iones hierro(II), una vez alcanzado el equilibrio; dicho resultado es similar al descrito por la isoterma tipo II.

El análisis comparativo de los resultados experimentales con las ecuaciones isotermas de adsorción de Freundlich, Langmuir, Langmuir-Freundlich (Figura 4) demostró que la primera ecuación isoterma describe con mayor exactitud la adsorción de los iones hierro(II) en la toba vítrea del yacimiento de Magueyal.

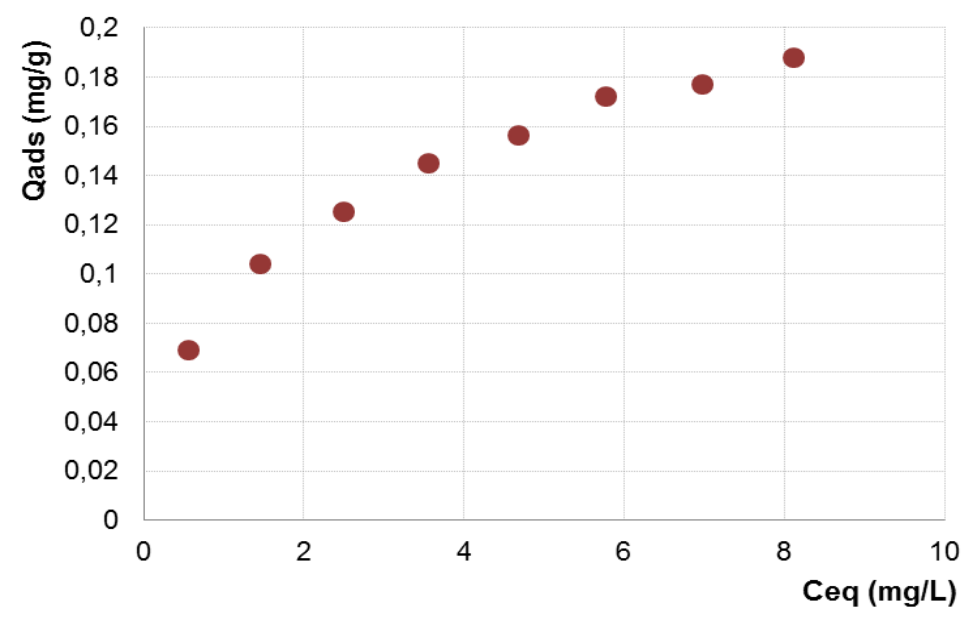

Figura 3. Isoterma de adsorción experimental de iones hierro(II) en el mineral de Magueyal en estado natural. 


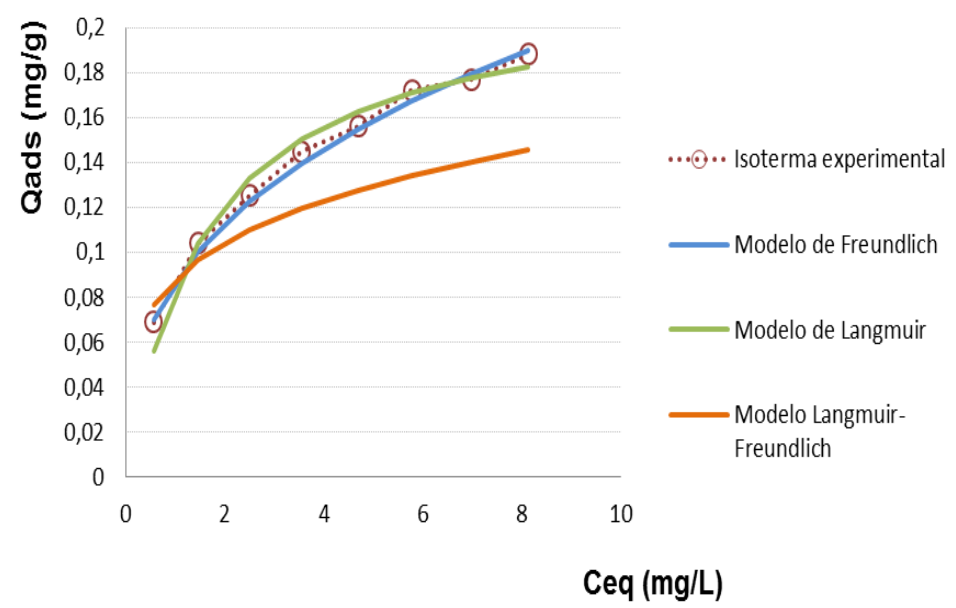

Figura 4. Isotermas de adsorción de hierro(II) en el mineral de Magueyal en estado natural.

Los parámetros de cada modelo para la adsorción de hierro(II) en el mineral de Magueyal en estado natural se indican en la Tabla 4. Los mejores resultados se obtuvieron ajustando la isoterma de adsorción experimental a la de Freundlich, lo que indica que la adsorción tuvo lugar en una superficie heterogénea del adsorbente, siendo posible la adsorción de varias capas del adsorbato. El factor de separación ( $R L$ ) calculado para el rango de concentración inicial del ión seleccionado para realizar el experimento alcanzó valores en el intervalo de 0 a 1 , lo que demuestra que la adsorción de iones hierro(II) en la toba vítrea es adecuada.

Tabla 4. Parámetros de las isotermas de adsorción de Langmuir, Freundlich y Langmuir-Freundlich

\begin{tabular}{ccc}
\hline Modelos & \multicolumn{3}{c}{ Parámetros } \\
\hline \multirow{2}{*}{ Langmuir } & $\mathrm{N}_{\mathrm{m}}(\mathrm{mol} / \mathrm{g})$ & 0,616 \\
& $\mathrm{~K}_{\mathrm{L}}$ & 0,135 \\
& $\mathrm{R}^{2}$ & 0,9579 \\
Freundlich & $\mathrm{K}_{\mathrm{F}}$ & 0,348 \\
& $1 / \mathrm{n}$ & 0,372 \\
& $\mathrm{R}^{2}$ & 0,9816 \\
Langmuir - Freundlich & $\mathrm{K}$ & 0,087 \\
& $\mathrm{~b}$ & $-0,013$ \\
& $\mathrm{n}$ & 4,255 \\
& $\mathrm{R}^{2}$ & 0,7768 \\
\hline
\end{tabular}

Los resultados son similares a los obtenidos en las investigaciones para la adsorción de iones cobre(II) en las tobas vítreas del yacimiento de 
Magueyal (Garrudo 2013; Salazar y Pérez 2015) y en la adsorción en dióxido de silicio amorfo sintético (Prieto-García et al. 2012). Además, se determinó que la capacidad máxima de adsorción del mineral estudiado fue de $34,4 \mathrm{mg} / \mathrm{g}$; siendo este un valor superior a los reportados para el carbón granular y la clinóptilolita e inferior en los casos de minerales modificados y al carbón activado.

Otro aspecto investigado en la eficiencia de recuperación de hierro(II) fue el efecto de la presencia de cobre(II) en la solución sintética, mediante la planificación de experimentos, considerando como variables independientes la concentración de cobre(II), la temperatura y la agitación. Se comprobó estadísticamente, con un nivel de confianza del $95 \%$, la influencia positiva de la concentración de cobre(II), mientras que el resto de las variables no son significativas; no así la combinación de los factores, siendo la de mayor influencia la combinación concentración de cobre(II) y la temperatura. La no influencia del cobre(II) en la remoción de hierro(II) es similar a otras investigaciones de remoción conjunta de iones metálicos (Alslaibi et al. 2014).

En la Tabla 5 se muestran los resultados de la remoción de hierro(II) en soluciones sintéticas y reales en la toba vítrea del yacimiento de Magueyal.

Tabla 5. Remoción de iones hierro(II) en soluciones sintéticas y reales en la toba vítrea

\begin{tabular}{ccc}
\hline $\begin{array}{c}\text { Tiempo de } \\
\text { contacto } \\
\text { (min) }\end{array}$ & $\begin{array}{c}\text { Remoción de iones } \\
\text { hierro(II) en soluciones } \\
\text { sintéticas (\%) }\end{array}$ & $\begin{array}{c}\text { Remoción de iones } \\
\text { hierro(II) del residual } \\
\text { real (\%) }\end{array}$ \\
\hline 60 & $36,48 \%$ & $29,4 \%$ \\
80 & $37,60 \%$ & $32,2 \%$ \\
100 & $37,68 \%$ & $33,5 \%$ \\
\hline
\end{tabular}

En las pruebas con soluciones reales para tiempos de contactos distintos, tal como se indica en la Tabla 5 , se comprobó el incremento de la remoción de iones hierro(II) hasta alcanzar el 33,5 \%, con respecto al tiempo de contacto. Estos resultados son similares a los obtenidos en la adsorción de iones hierro(II) cuando se utilizan soluciones sintéticas, lo que demuestra que la presencia de otros iones no afecta considerablemente la selectividad del adsorbente por los iones en estudio, en la solución residual real.

\section{CONCLUSIONES}

- La cinética del proceso de remoción de los iones hierro(II) se describe por el modelo de pseudoprimer orden, siendo la difusión externa la etapa limitante. 
- La isoterma de adsorción experimental se corresponde con el tipo II y el mejor ajuste se logra con el modelo de Freundlich; se alcanza la máxima capacidad de adsorción de $34,4 \mathrm{mg} / \mathrm{g}$, para los iones hierro(II).

- Los resultados obtenidos en la remoción de iones hierro(II), mediante el método de adsorción con el empleo de la toba vítrea del mineral de Magueyal es adecuado, ya que se logra disminuir el impacto ambiental negativo del residual líquido producido en las calderas de las termoeléctricas durante la limpieza química de las mismas.

\section{REFERENCIAS}

Albadarin, A. B.; Mangwandi, C.; Ala'a, H.; Walker, G. M.; Allen, S. J. y Ahmad, M. N. 2012: Kinetic and thermodynamics of chromium ions adsorption onto low-cost dolomite adsorbent. Chemical Engineering Journal, 179: 193-202.

Alslaibi, T. M.; Abustan, I.; Ahmad, M. A. y Foul, A. A. 2014: Kinetics and equilibrium adsorption of iron (II), lead (II), and copper (II) onto activated carbon prepared from olive stone waste. Desalination and water treatment, 52(40-42): 7887-7897.

Azizian, S. 2004: Kinetic Models of Sorption a Theoretical Analysis. Journal of colloid and Interface Science, 276(1): 47-52.

Batista, W. E. 2016: Empleo de minerales cubanos modificados Magueyal y Samá Arriba para la disminución de cobre(II) en aguas residuales. Instituto Superior Politécnico José Antonio Echeverría.

Blanco-Flores, A.; Autie-Castro, G.; Rodríguez-Montes de Oca, D.; RicardoPáez, S.; López-Cordero, R. y Autie-Pérez, M. 2009: Características superficiales del vidrio volcánico y remoción de Cu2+ desde disoluciones acuosas. Revista Latinoamericana de Recursos Naturales, 5(3): 238252.

Córdova, V. 2015: Potencialidades de la zeolita natural del yacimiento de Palmarito de Cauto para el tratamiento de licores básicos que contienen cromo. Universidad de La Habana. Tesis doctoral. 125 p.

Fernández-Hechevarría, H.; A-Cecilia, J.; Garrudo-Guirado, M. I.; LabadieSuarez, J. M.; Contreras-Larios, J. L.; Autie-Pérez, M. A.; RodríguezCastellón, E. 2016: Characterization and use of a cuban mineral in elimination of crystal violet from aqueous solution. International Journal of Plant, Animal and Environmental Science, 6(2): 332-46.

Garrudo, M. I. 2013: Utilización de una toba vítrea cubana modificada para la disminución de la concentración de cobre(II) en aguas residuales. Universidad Tecnológica de La Habana. 
Ghassabzadeh, H.; Mohadespour, A.; Torab-Mostaedi, M.; Zaheri, P.; Maragheh, M. G. y Taheri, H. 2010: Adsorption of Ag, $\mathrm{Cu}$ and $\mathrm{Hg}$ from aqueous solutions using expanded perlite. Journal of hazardous materials, 177(1-3): 950-955.

Keng, P. S.; Lee, S. L.; Ha, S. T.; Hung, Y. T. y Ong, S. T. 2014: Removal of hazardous heavy metals from aqueous environment by low-cost adsorption materials. Environmental Chemistry Letters, 12(1): 15-25.

Machado-Infante, J.; Ramírez-Caballero, G. y Barajas-Meneses, M. 2016: Study of the adsorption capacity of Fe (II) dissolved in water by using a mineral rich in Manganese Dioxide (MnO2) from Colombia. Dyna, 83(196): 223-228.

Nibou, D.; Mekatel, H.; Amokrane, S.; Barkat, M. y Trari, M. 2010: Adsorption of $\mathrm{Zn}^{2+}$ ions onto $\mathrm{NaA}$ and $\mathrm{NaX}$ zeolites: Kinetic, equilibrium and thermodynamic studies. Journal of Hazardous Materials, 173(1-3): 637-646.

Organismo Nacional de Normalización de la República de Cuba Oficina Nacional de Normalización (ONN). NC 521: 2007: Vertimiento de aguas residuales a la zona costera y aguas marinas-especificaciones. La Habana.

Plazinski, W.; Dziuba, J. y Rudzinski, W. 2013: Modeling of sorption kinetics: the pseudo-second order equation and the sorbate intraparticle diffusivity. Adsorption, 19(5): 1055-1064.

Prieto-García, J. O.; Quintana-Puchol, R.; Santana-Rodríguez, A.; Echevarría-Bermúdez, Y.; Treto-Suarez, M. y Mollineda-Trujillo, Á. 2012: Estudio cinético y termodinámico de la adsorción de iones cobre(II) en un adsorbente de base silícica. Revista Cubana de Química, XXIV(1): 40-47.

Salazar, M. y Pérez, F. 2015: Remoción de cobre(II) en medio acuoso empleando una arcilla cubana natural y modificada. Universidad Tecnológica de La Habana.

Toor, M. y Jin, B. 2012: Adsorption characteristics, isotherm, kinetics, and diffusion of modified natural bentonite for removing diazo dye. Chemical Engineering Journal, 187: 79-88.

Vera-Alatrista, C. H. 2006: Capacidad de adsorción de contaminantes químicos de aguas residuales utilizando piedra pómez. Universidad Nacional del Altiplano. Perú. Tesis de maestría. 134 p. Consultado: 15/04/2018. Disponible

en: http://repositorio.unap.edu.pe/handle/UNAP/582

Recibido: $22 / 11 / 2018$

Aceptado: 09/01/2019 